\title{
Evolution of reconstructive techniques following endoscopic expanded endonasal approaches
}

\author{
Amin Kassam, M.D., Ricardo L. Carrau, M.D., Carl H. Snyderman, M.D., \\ Paul Gardner, M.D., AND Arlan Mintz, M.Sc., M.D. \\ Departments of Neurological Surgery and Otolaryngology, Minimally Invasive Neurosurgery Center \\ University of Pittsburgh; and University of Pittsburgh Medical Center, Presbyterian University \\ Hospital, Pittsburgh, Pennsylvania
}

\begin{abstract}
Harvey Cushing first popularized the transsphenoidal route to the sella turcica, and Jules Hardy subsequently refined it by adding the operating microscope. Over the ensuing decades, attempts at extending the application of this approach have been advanced by Edward Laws and others. With the evolution of endoscopic approaches, the natural expansion of their use to intradural lesions followed. For the expanded endonasal approach to become a viable option, the paramount concerns surrounding consistent reconstruction of the dura mater must be overcome. In this review the authors chronicle the evolution of the reconstruction technique they currently use after performing expanded endonasal approaches. They also report the use of a balloon stent to buttress the reconstruction and counter the effects of graft migration and cerebrospinal fluid fistula formation. The technique described in this report represents an important step forward in the reconstruction of defects resulting from expanded endonasal approaches.
\end{abstract}

\section{KEY WORDS • cerebrospinal fluid leak • endoscopy • endonasal approach • transsphenoidal approach $\bullet$ reconstruction}

Effective and consistent reconstruction of the dura mater has been a major challenge that has significantly hindered the extension of transsphenoidal approaches to regions beyond the paranasal sinuses. For these approaches to be applied fully to the intracranial cavity and become viable options for intradural tumor removal, this issue must be resolved. Over the past decade, significant anatomical and instrumentation advances have been made, facilitating the exposure and resection of intradural lesions via a fully endoscopic expanded endonasal approach. In this report we describe our progressive experience with reconstruction of the defects that ensue from this approach. Our learning curve is described, from our initial reconstructive technique to the approach we currently use, and the lessons learned over the past 7 years are detailed.

The goals in reconstructing defects resulting from the expanded endonasal approach are identical to those of reconstruction after conventional external approaches; that is, to separate the cranial cavity completely from the sinonasal tract, obliterate the dead space, and preserve the neurovas-

Abbreviation used in this paper: $\mathrm{CSF}=$ cerebrospinal fluid. cular and ocular function. ${ }^{5}$ The underlying principle of multilayered reconstruction to reestablish tissue barriers is also the same.

\section{SURGICAL TECHNIQUE}

\section{Preoperative and Intraoperative Considerations}

Preoperative considerations are similar for all reconstructive techniques. Factors such as the area and volume of the resection, extent of the communication between the cranial cavity and the upper aerodigestive tract, adequacy and geometry of the remaining dural and bone margins, general status of the patient, previous intranasal or maxillofacial surgeries, and the possibility of increased CSF pressure postoperatively have to be considered for the reconstruction. A variety of options such as free tissue grafts, vascularized local flaps, free microvascular flaps, and allografts are available for this purpose; however, to date we have relied exclusively on the use of free tissue grafts. There are no viable strategies for microvascular tissue flaps currently available, but these are being actively developed. 


\section{Multilayered Reconstruction}

Initially our reconstructive technique represented an intuitive extension of our experience with the endoscopic repair of CSF leaks as well as our experience with reconstruction of the skull base after oncological resections performed using traditional external approaches. ${ }^{3-8}$ Specifically, we adhered to the general principles of multilayered reconstruction. Whenever possible we began with an epidural inlay graft, that is, one placed between the dura mater and the skull base. Unfortunately, this is only feasible in a limited number of defects in which the graft can be safely tucked under the remaining bone ledge. This often proves to be a difficult task, particularly in defects without an adequate bone ledge due to the proximity of neurovascular structures, such as defects of the planum sphenoidale or the lateral sphenoid wall adjacent to the internal carotid artery or optic nerve. As a consequence, the inlay graft is not always possible. The next layer consists of an onlay graft, placed extracranially between the skull base and the sinonasal tract. The ensuing layer is a fat graft, which is reserved for the obliteration of bone cavities such as the sphenoid sinus or its lateral recess. We have not used intradural fat grafts because of our concern about potentially recreating the mass effect associated with the initial tumor. Although localized vascularized flaps such as middle turbinate flaps are available, we rarely use these. In our experience the pedicle and geometry of these flaps tend to pull them away from the skull base defect, and in addition, their lining is frequently sacrificed during the exposure to provide adequate access or is involved with tumor and requires resection.

Our experience with alloplastic materials such as bone cements during external approaches yielded disappointing long-term results because of a high incidence of infection and sequestrum formation. These concerns were further magnified during our initial attempts to use these materials after performing an expanded endonasal approach. We therefore strongly discourage the use of these substances. The use of free bone grafts to reinforce the sella turcica after performing microseptal transsphenoidal approaches has been popularized over the last few decades. During expanded endonasal approaches we found this to be infeasible given the extent of the majority of defects created and the concerns related to positioning the graft epidurally, which have been previously discussed.

\section{Initial Reconstructive Technique}

The defects that we were creating with the different modules of the expanded endonasal approach were reconstructed by reestablishing a multilayer barrier with free tissue grafts. As discussed, we initially applied the technique for spontaneous CSF leaks. ${ }^{7,8}$ Specifically, we started by using cadaveric pericardium and placing it as an inlay graft in the epidural space (between the dura mater and the bone at the cranial base) when feasible. Given the difficulty accomplishing this, the graft was therefore tucked under as many points as possible to seal the defect and minimize the risk of migration. This not only proved to be a tedious process but offered little stability.

The next layer of the barrier, an onlay graft, was then placed around and overlapping the bone defect. Initially we applied a layer of fibrin sealant between the inlay and onlay grafts to minimize the risk of graft migration. Nevertheless, we subsequently found that this layer of fibrin was counterproductive because it prevented direct contact of the grafts and often seeped under them, creating not only separations between the two layers but also between the grafts and the bone, thus preventing healing. Clinically, this resulted in a number of leaks that developed at approximately 2 weeks postoperatively, corresponding to the time of degradation and reabsorption of the fibrin sealant.

Following placement of the onlay graft, the next layer consisted of free abdominal fat to provide a biological dressing and further promote vascularized granulation tissue formation. This fat plug also has a tamponade effect, applying pressure on the inlay/onlay grafts. Fibrin sealant was applied to help stabilize and seal the margins of the fat graft (Fig. 1). We then placed sponge packing to further hold the fat in place (Video 1; Click here to view with Windows Media Player and a braodband connection, here with a dial-up connection, on here to view with RealPlayer.). During this early period in our reconstruction experience we routinely placed a lumbar drain for 3 to 5 days to minimize CSF pressures, believing that this would reduce the risk of dislodging the graft and minimize the risk of creating CSF fistulas.

This sequence represented our reconstruction technique for several years. Unfortunately, this initial technique extrapolated from our spontaneous CSF leak repair experience proved to be problematic when applied to reconstruction after expanded endonasal approaches. The incidence of CSF leaks following expanded endonasal approaches proved to be a significant challenge (Kassam, et al., unpublished data). Nevertheless, despite the large number of leaks needing revision, the secondary morbidity and sequelae were not clinically significant. Specifically, during this initial experience the incidence of culture-positive bacterial meningitis in the first 400 cases was less than 3\%. Nevertheless, leaks proved to be a source of frustration and extended the length of hospital stay and periodically resulted in readmission of some patients. Notably, over this time period we did not require a transcranial approach to seal any of the defects, and all were resolved via an endonasal reexploration and augmentation of the graft.

\section{Lessons Learned}

This proved to be an exceptionally valuable learning experience because during these reexplorations we observed several key factors. The most common finding was either the development of a small persistent channel of CSF at the most dependent site (Fig. 2), or migration of the graft away from the bone margin of the defect superiorly (Fig. 3). We further observed that after an expanded endonasal approach, CSF leaks varied significantly based on the body habitus of the patient, the disease being treated, the site of the surgery, and whether arachnoid cisterns (and occasionally ventricles) needed to be opened. Defects in the anterior cranial base were much more likely to leak than clival defects. We postulated that this might be related to the effects of gravity during upright positioning, making the grafts more vulnerable to separation.

The size of the tumor did not correlate with the incidence of CSF leakage after an expanded endonasal approach. Empirically we observed that opening the arachnoid cisterns widely, as is often required for resection, proved to be the 

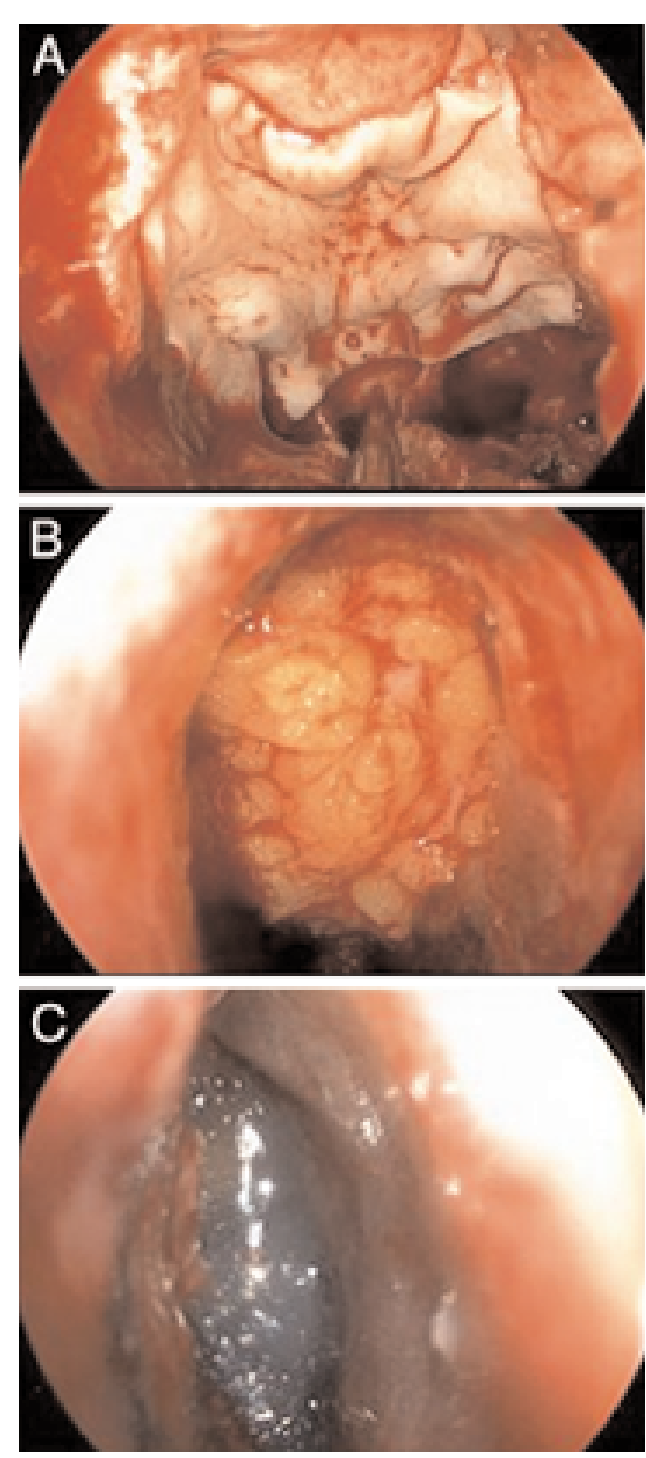

Fig. 1. Composite of intraoperative photographs acquired through the endoscope, showing the key steps of the initial reconstructive technique used after an expanded endonasal approach. A: The position of the onlay graft is depicted with redundancy across the bone margin of the defect. B: Obliteration of the sphenoid sinus by the fat graft is shown. C: The fibrin plug used to create a coagulum and pressure in hopes of stabilizing the fat graft is depicted.

factor most consistently predicting the likelihood of CSF leakage. This was most evident in our craniopharyngioma series (Kassam, et al., unpublished data). The majority of these lesions were small but required extensive intraarachnoidal dissection and often endonasal third ventriculostomies. Based on these observations we believe that opening a cistern created a path of least resistance for the passage of CSF through the defect created by the expanded endonasal approach, therefore increasing the risk of graft migration and formation of a CSF fistula, which created a persistent channel (Fig. 2). A second surgery was then needed to repair the persistent fistula, and occasionally this entity required permanent CSF diversion.

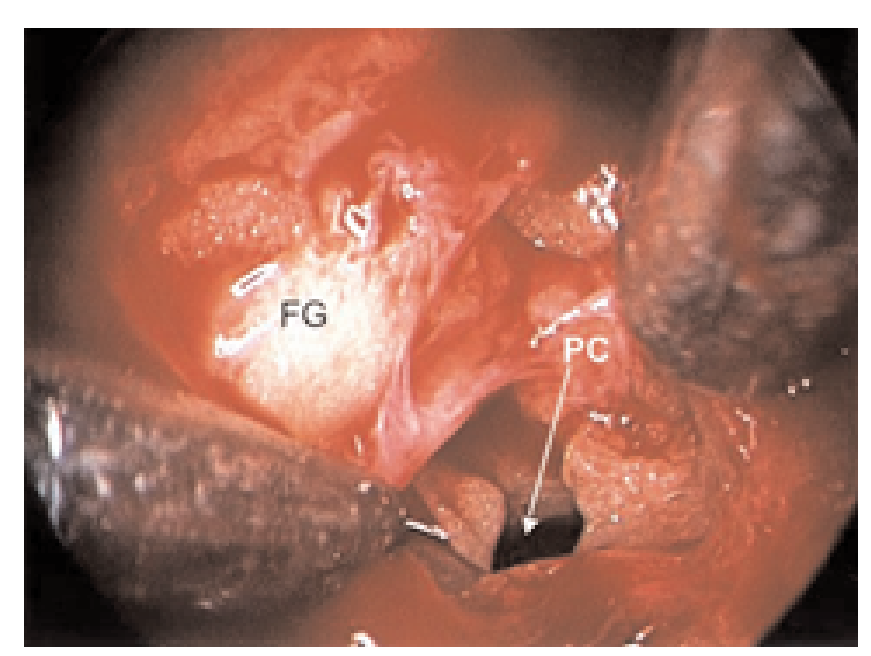

Fig. 2. Endonasal view obtained during reexploration for a CSF leak that arose after an expanded endonasal approach, demonstrating that the majority of the graft has granulated well. The fat graft (FG) can be seen forming the granulation defect. A small persistent channel (PC) can be seen inferiorly at the most dependent site. This represents a relatively small defect when compared with the No. 6 French suction tube seen in the photograph. Note the significant separation between the edges of the granulation tissue.

During reexplorations, we invariably found that most of the margins of the graft had sealed but there was a single and often small area that had not healed. This area was usually located at the most dependent site, inferiorly at the point of maximum pressures, or else superiorly at the site at which the graft is most vulnerable to migration (Fig. 3). Under these circumstances we found it unnecessary to remove the part of the graft that had healed, thereby restarting the entire process. Instead, we removed only the segments of the overlying graft that had not sealed and followed the flow of CSF to the persistent channel. Once the fistula site was identified we repaired the defect with a new inlay and/or onlay graft, followed by repacking with abdominal fat. This was adequate in most instances. In case of significantly elevated CSF pressure and persistent fistula, which was most evident with craniopharyngiomas, a permanent CSF diversion was needed.

Based on these observations it became apparent that failures resulted from the development of persistent channels that formed within the reconstructed area and/or graft migration. We further postulated that the greatest threat to the graft likely occurred very early, perhaps during the extubation sequence, when unavoidable straining results from Valsalva maneuvers. We also believed that the graft was most vulnerable to this early failure if the arachnoid had been opened widely and therefore was no longer able to offer resistance and protect the graft. During our very early experience, we treated postprocedure leaks with lumbar drains to divert CSF in hopes of promoting healing. This proved to be futile, however, in the overwhelming majority of cases. Based on our observations during reexplorations, we believed this failure was due to the significant fistula that had been created, thus precluding contact of the residual tissue required for vascularization. Specifically, we believed that despite CSF diversion it would be most un- 


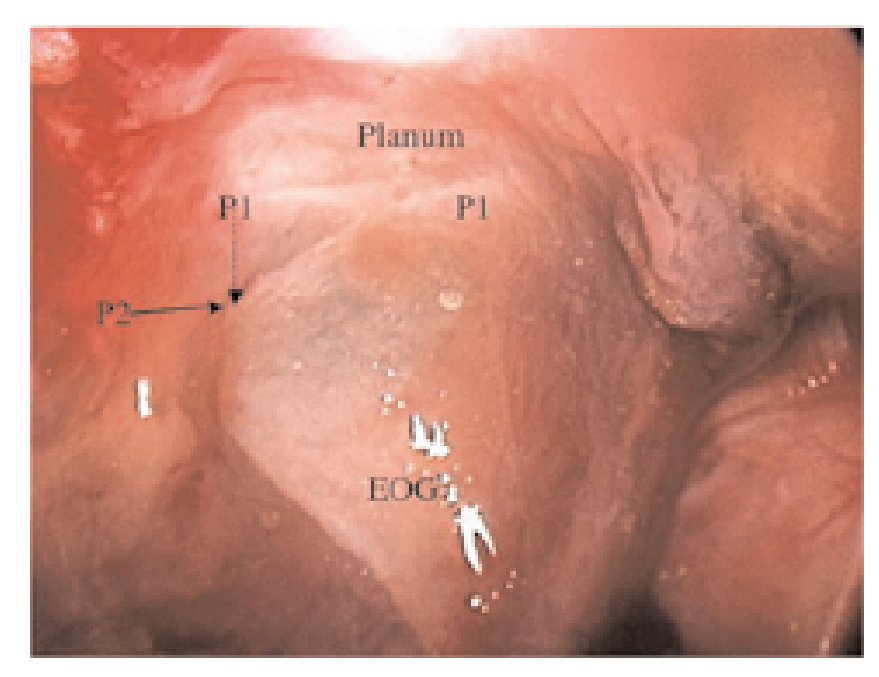

Fig. 3. Endonasal view obtained during reexploration for CSF leakage that occurred as a result of an expanded endonasal approach following an earlier transplanum approach. This photograph shows migration of the extradural onlay graft (EOG), which was placed along the planum sphenoidale. The graft is maintained in its original position (P1) on the patient's left side; however, it has migrated (dashed arrow) to a more caudal position (P2, solid arrow) on the patient's right.

likely that the tissue margins would collapse enough to develop contact and adherence.

\section{Current Technique}

Based on these observations, we have made several critical modifications to our initial technique. We changed the choice and position of the graft materials, and we now place an initial subdural inlay graft between the brain and the dura mater (Fig. 4). We favor the use of collagen matrix (Duragen; Integra Life Sciences, Plainsboro, NJ) because it affords the best tissue-handling properties as an inlay graft. This subdural graft should be slightly redundant to extend beyond the dural margins, ideally 5 to $10 \mathrm{~mm}$ in all directions (Fig. 4). The pliability of this collagen matrix allows for gentle manipulation around critical neurovascular structures, avoiding the difficulties noted earlier when positioning inlay grafts. Based on our findings, Duragen should not be used in isolation as a graft because it does not offer the resistance to CSF that is required and will leak through the center of the graft even if the edges have sealed. Therefore, a subsequent onlay graft is placed epidurally on top of the Duragen inlay and extends to overlap along the bone margins of the defect (Fig. 5).

We have adopted the use of acellular dermis as our material of choice for the onlay graft. Although it is important to point out that this is an off-label indication, we have found that its handling characteristics, its availability (there isno need for harvesting from a distant site), and its remarkably rapid epithelialization and ingrowth of fibrous tissue are valuable in preventing graft migration/early separation while promoting healing. ${ }^{1,2}$ We prefer the use of moderatethickness grafts $(0.3-0.7 \mu)$ because this material offers the best tissue-handling properties. The graft should be adequately presoaked in saline to optimize its handling. It is also imperative to use a single graft that is redundant

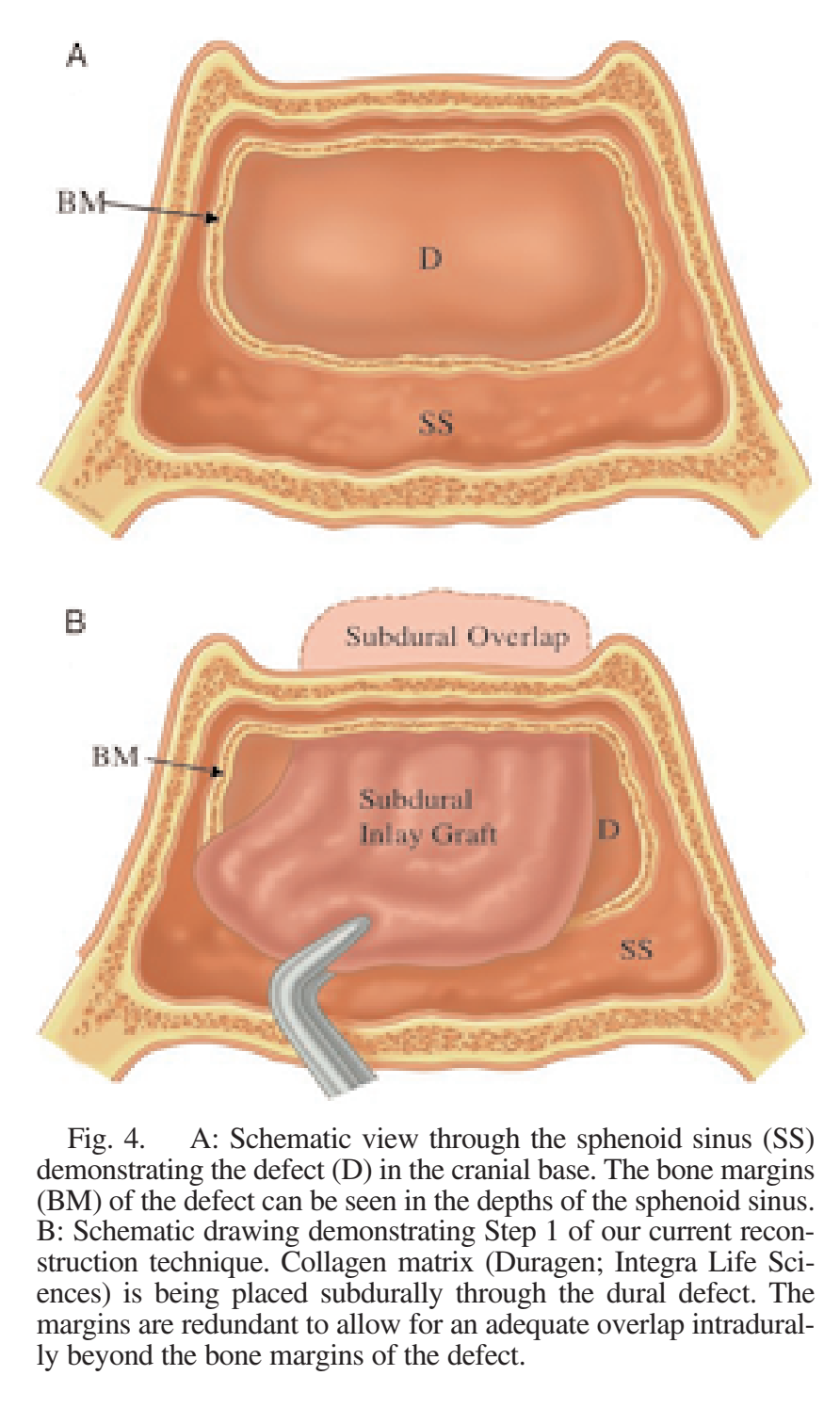

to extend beyond the margin of the bone defect (Fig. 5). Before placing the graft, all the mucosa must be removed from the bone onto which the onlay graft is placed, to permit the critical direct contact that is required for revascularization. Early in our experience we used multiple small pieces of grafts, and this led to separation and crevices between the pieces that resulted in persistent CSF channels. For this reason it is imperative that the single graft when placed have no folds within it and that it is completely flat, allowing maximum bone contact and reducing the risk of channel formation.

At this stage the paranasal sinus is obliterated by fat harvested in the patient. During placement of the fat there is a significant risk of shifting the underlying inlay/onlay grafts (Fig. 6). In general we are much more vigilant to avoid this shifting of the underlying tissue barriers as we place each one over the other. The fat not only provides a biological dressing, promoting vascularization and healing, but also applies pressure, reducing the risk of migration of the underlying grafts. Fibrin sealants are no longer used between the inlay and onlay grafts, but rather are applied only after 


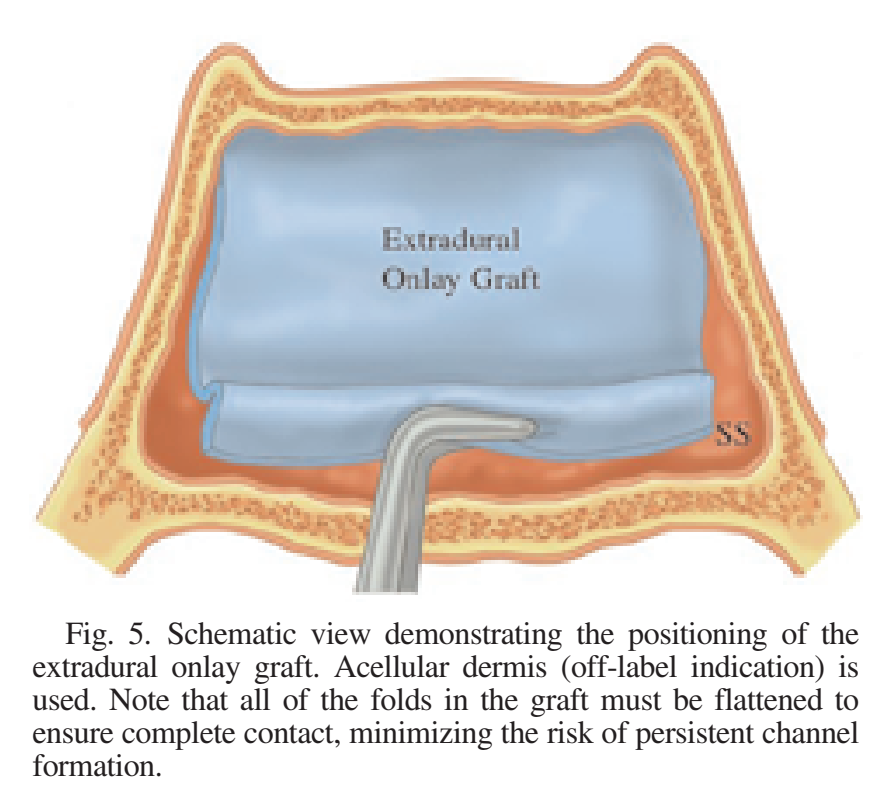

the final tissue barrier (that is, fat) has been positioned within the other paranasal sinus (Fig. 7).

To mitigate the effects of graft migration and the development of persistent channels, we now use a balloon stent to apply pressure against the fat graft (Fig. 8). This represents our most recent modification, and perhaps the most important one, because this allows gentle pressure to be applied against the fat graft, which in turn stabilizes the inlay/onlay grafts, minimizing migration. Furthermore, we opine that the increased resistance offered by the balloon minimizes the development of early persistent CSF channels. At present we are using a No. 12 French Foley balloon catheter that is passed from the left nares across the residual portion of the nasal septum, and the distal portion is placed into the upper nasopharynx across the midline (Figs. 8 and 9). This allows the balloon to be wedged between the residual nasal septum and the fat graft, thus minimizing the risk of migration (Video 2; Click here to view with Windows Media Player and a braodband con-

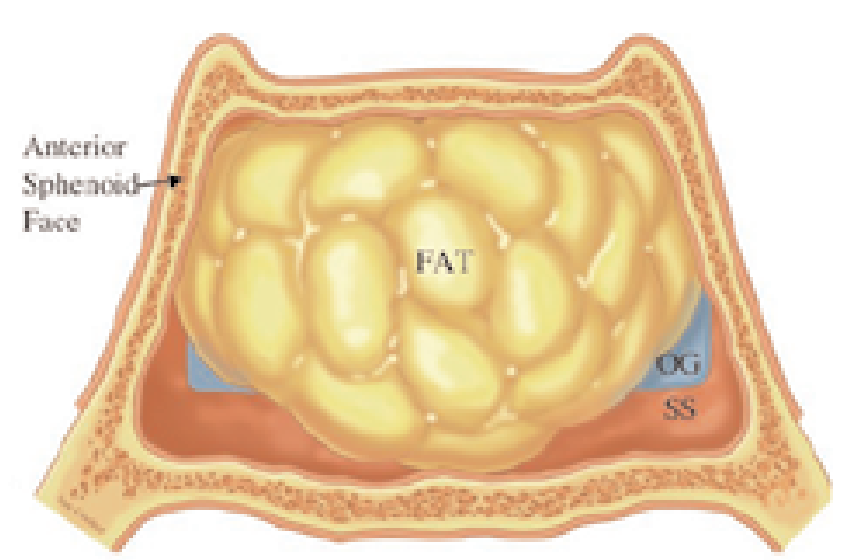

Fig. 6. Schematic view demonstrating the placement of the fat graft on top of the epidural onlay graft (OG). This obliterates the sphenoid sinus and provides pressure on the onlay graft beneath.

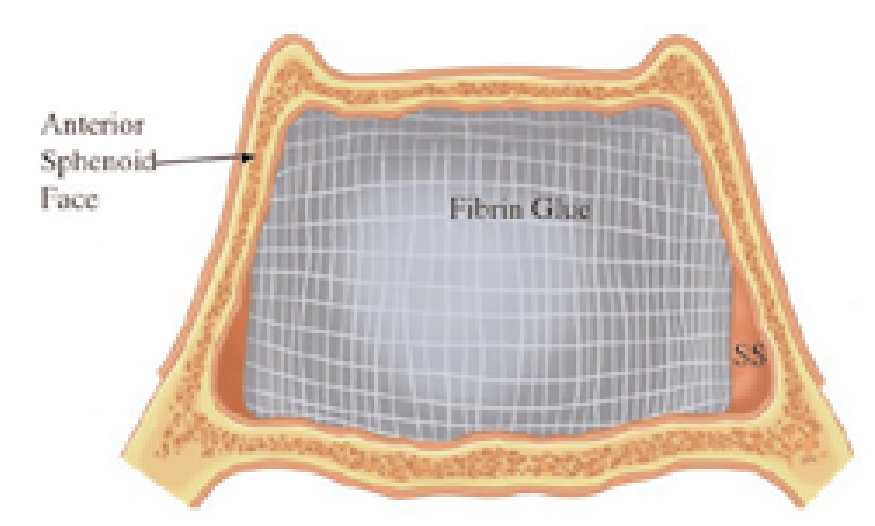

Fig. 7. Schematic view showing the placement of fibrin glue on top of the fat graft to create a coagulum to the level of the anterior face of the sphenoid sinus.

nection, here with a dial-up connection, or here to view with RealPlayer.).

The fat is used to obliterate the sphenoid sinus and the balloon must be placed external to the sinus to avoid compression and injury of any exposed neurovascular structures (Fig. 8). In effect, the balloon recreates the sphenoid face. The placement and inflation of the balloon catheter is carefully performed under endoscopic vision by using a maximum of $5 \mathrm{ml}$ of saline. Overinflation may result in compressive effects and injury, and the patient should be examined daily to ensure that these are not occurring. The balloon is left in place for 3 to 5 days.

Another advantage of the balloon is afforded by the surgeon's ability to deflate it prior to removal. This prevents the negative pressure that can be created when removing other forms of nasal packing such as sponges. The presence of negative pressure can potentially dislodge the underlying grafts. Use of the balloon has reduced the incidence of CSF

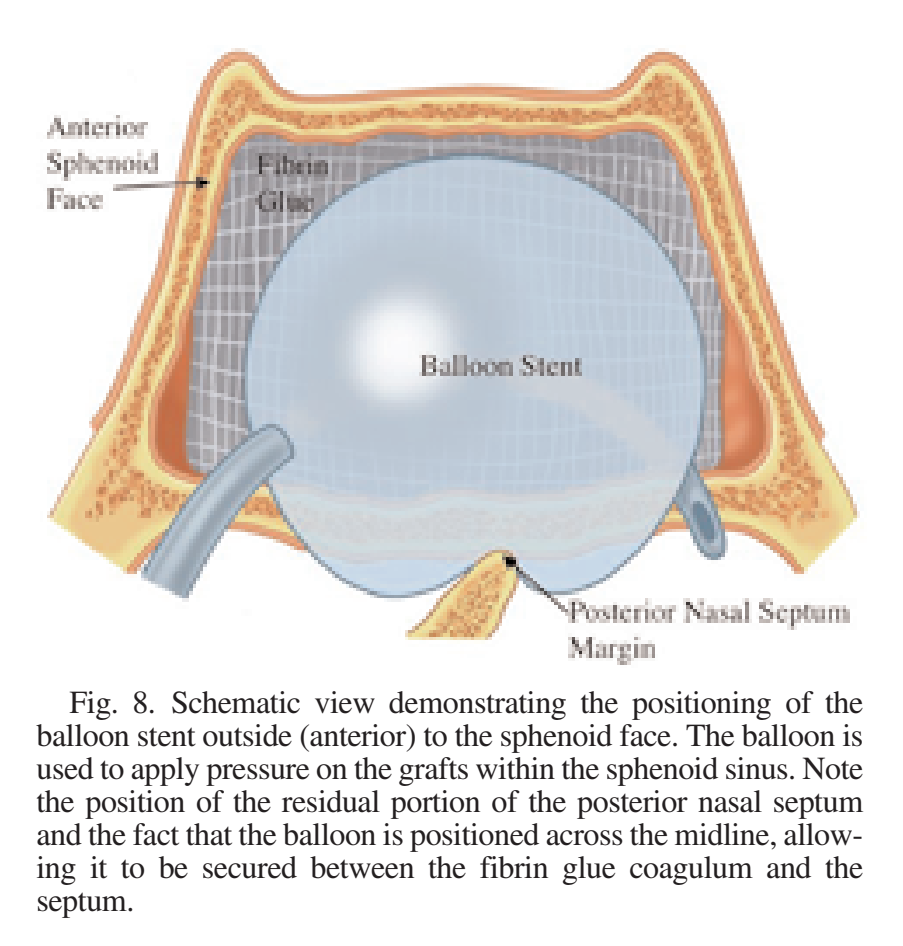




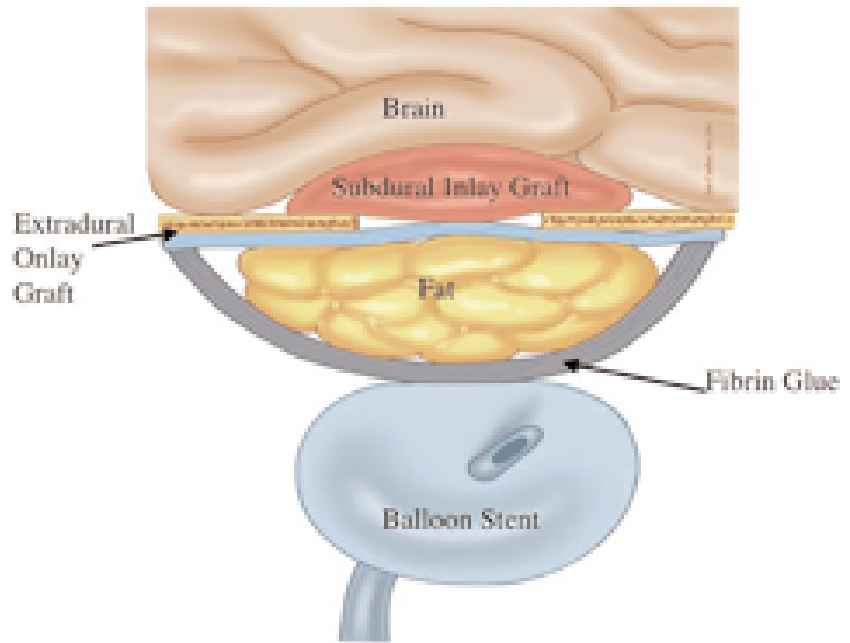

Fig. 9. Schematic view demonstrating the current multilayered reconstruction technique. Note the redundancy of the subdural inlay graft (shown in red) allowing for contact with the residual dura mater. The pressure applied by the brain further promotes this contact and obliterates the subdural dead space. Similarly, note the redundancy in the onlay graft (shown in blue) with complete bone contact. Note the resistance of the balloon, which acts to minimize graft migration.

leaks following the expanded endonasal approach; however, ideal geometries and configurations of balloon shape, pressure, and inflation still need to be developed.

Finally, we no longer routinely place a lumbar drain at the time of surgery. We have found this practice to be counterproductive because it potentially creates negative pressure that may result in separation of the initial subdural inlay graft from the dural edges.

\section{Postoperative Management}

We recommend that the general principles of managing a CSF fistula be observed, including adjunctive measures to facilitate unhindered healing of the defect; these include avoiding activities that raise the intracranial pressure (for example, straining, leaning forward, or lifting weights heavier than $15 \mathrm{lbs}$ ). Other measures include use of stool softeners, sneezing with an open mouth, and avoidance of nose blowing.

The use of prophylactic antibiotic medications for the prevention of meningitis in patients with CSF fistulas continues to be controversial. We believe strongly, however, in using a third-generation cephalosporin administered at the time of surgery. We then continue antibiotic therapy while the balloon is in place. Although there is no rigorous evidence to indicate that this is of value, we believe it is important to prevent colonization of the wound and potential transmission of infection to the CSF.

A postoperative computerized tomography scan or magnetic resonance image obtained within the first 24 hours after surgery is important to rule out evidence of intracranial bleeding, parenchymal injury, or tension pneumocephalus. We favor routine imaging of the brain, even in the absence of a neurological deficit. This is also of value to confirm the ideal positioning of the balloon. The balloon is usually re- moved between Days 3 and 5, depending on risk factors such as the degree of arachnoid dissection and the patient body habitus. This is usually done in an outpatient setting by using nasal endoscopy, and the nasal passages are debrided of any clot and debris at the same time.

\section{DISCUSSION}

Since the inception of this current reconstruction strategy, several of the modifications described previously have completely obviated the need for permanent diversion of CSF after an expanded endonasal approach. In particular, the use of the subdural inlay graft to obliterate the dead space in an attempt to recreate the resistance offered by the violated arachnoid is critical. The avoidance of fibrin sealants between the inlay/onlay to allow for unabated contact of the granulation tissue is also important. Similarly, although it is counterintuitive, the avoidance of routine lumbar drainage that can potentially result in separation of the subdural inlay graft from the dural margins, and use of a balloon instead to offer increased resistance has been an important step forward.

There remain significant challenges, because the shape of the balloon is currently less than ideal. It needs to be better contoured to the skull base to maximize the resistance in a safe manner. The rare situation in which the current technique has failed has been at the site where the balloon did not make adequate contact with the graft as a result of its spherical shape. The other source of failure is the use of preoperative radiation and chemotherapy, which can significantly compromise wound healing and the formation of stable granulation tissue. As a response, we are currently developing a microvascular free flap designed for expanded endonasal approaches.

\section{CONCLUSIONS}

During our 7-year experience with the expanded endonasal approach, we initially expended a significant amount of effort and resources focusing on the approach and resection phase. As a consequence, a number of biotechnological innovations were achieved to facilitate these components of the procedure. Over this time period we were able to define the nature of the ensuing defects and establish the parameters that would be required for successful reconstruction. Learning from these experiences, we have developed our current technique, which has at this very preliminary phase demonstrated initial efficacy. If the expanded endonasal approach is truly to be established as a viable approach for centrally located intradural lesions, the longterm effectiveness of this reconstructive technique will have to be clearly established. Having established the feasibility of approaching and resecting appropriately selected cases via the expanded endonasal approach, we are confident that the increased focus on reconstruction will yield emerging biotechnologies that will further refine our present technique.

\section{References}

1. Buinewicz B, Rosen B: Acellular cadaveric dermis (AlloDerm): 


\section{Reconstruction after endoscopic expanded endonasal approach}

a new alternative for abdominal hernia repair. Ann Plast Surg 52: 188-194, 2004

2. Butler CE, Prieto VG: Reduction of adhesions with composite Alloderm/polypropylene mesh implants for abdominal wall reconstruction. Plast Reconstr Surg 114: 464-473, 2004

3. Hegazy HM, Carrau RL, Snyderman CH, et al: Transnasal endoscopic repair of cerebrospinal fluid rhinorrhea: a meta-analysis. Laryngoscope 110: 1166-1172, 2000

4. Kassam A, Carrau RL, Horowitz M, et al: The role of fibrin sealants in cranial base surgery. (http://www.medscape.com/ viewprogram/1280) [Accessed 28 June 2005]

5. Neligan PC, Mulholland S, Irish J, et al: Flap selection in cranial base reconstruction. Plast Reconstr Surg 98: 1159-1166, 1996

6. Snyderman CH, Janecka IP, Sekhar LN, et al: Anterior cranial base reconstruction: role of galeal and pericranial flaps. Laryngoscope 100: 607-614, 1990

7. Zweig JL, Carrau RL, Celin SE, et al: Endoscopic repair of acquired encephaloceles, meningoceles, and meningo-encephaloceles: predictors of success. Skull Base Surg 12: 133-140, 2002

8. Zweig J, Carrau RL, Celin SF, et al: Endoscopic repair of cerebrospinal fluid leaks to the sinonasal tract: predictors of success. Otolaryngol Head Neck Surg 123: 195-201, 2000

Manuscript received May 31, 2005.

Accepted in final form June 28, 2005.

Address reprint requests to: Amin Kassam, M.D., University of Pittsburgh School of Medicine, 203 Lothrop Street, Suite 500, Pittsburgh, Pennsylvania 15213. email: kassamab@upmc.edu. 